Check for updates

Cite this: RSC Adv., 2018, 8, 20450

\title{
Mechanical properties of epoxy nanocomposites filled with melamine functionalized molybdenum disulfide $\dagger$
}

\author{
Bin Chen, ${ }^{a}$ Bao-Jian Ni, ${ }^{a}$ Wen-Tao Liu, ${ }^{\text {b }}$ Qiu-Yang Ye, ${ }^{\text {b }}$ Si-Yuan Liu, \\ He-Xin Zhang (D) ${ }^{* b c}$ and Keun-Byoung Yoon*c
}

In this work, a melamine functionalized molybdenum disulfide $\left(\mathrm{M}-\mathrm{MOS}_{2}\right)$ was prepared and used as fillers to form epoxy (EP)/MoS 2 nanocomposites. The effects of molybdenum disulfide $\left(\mathrm{MoS}_{2}\right)$ and melamine functionalized molybdenum disulfide $\left(M-\mathrm{MoS}_{2}\right)$ loading on the mechanical properties of epoxy composites were investigated and compared. With only addition of $0.8 \mathrm{wt} \% \mathrm{M}-\mathrm{MoS}_{2}$, the tensile strength and modulus of EP/M-MoS 2 nanocomposites showed 4.5 and 4.0 times increase over the neat epoxy. Interestingly, the elongation at break value of EP was also increased with the introduction of $M-\mathrm{MoS}_{2}$ fillers. These properties could result from the good dispersion and strong interfacial adhesion of $M-M_{0} S_{2}$ fillers and the EP matrix. Therefore, this work provides a facile way to produce of high-performance EP nanocomposites.

Received 28th March 2018

Accepted 30th May 2018

DOI: $10.1039 / \mathrm{c} 8 \mathrm{ra0} 2689 \mathrm{k}$

rsc.li/rsc-advances

such as polystyrene, poly(methyl methacrylate), poly(vinylidene fluoride), polyvinyl alcohol, polyethylene and polypropylene. ${ }^{34-39}$ The resultant $\mathrm{MoS}_{2}$-filled polymer nanocomposites exhibited enhanced thermal stability, flame retardance and mechanical properties. With regards to EP resin, Y. Hu and Z. Gui et al. reported a $\mathrm{MoS}_{2}$-carbon nanotube reinforced EP composites. ${ }^{40}$ With the introduction of $2 \mathrm{wt} \% \mathrm{MoS}_{2}$-carbon nanotube, the organic volatiles and carbon monoxide was suppressed, while the mechanical properties were improved. N. Koratkar et al. also found the addition of exfoliated $\mathrm{MoS}_{2}$ could enhance the mechanical properties of EP resin. ${ }^{41}$ Although the $\mathrm{MoS}_{2}$ well dispersed in the EP matrix, the interfacial adhesion between the $\mathrm{MoS}_{2}$ and the EP matrix are less considered.

Therefore, in this research, we report a melamine functionalized $\mathrm{MoS}_{2}$ and further used as fillers to reinforce the EP resin. The functionalization of $\mathrm{MoS}_{2}$ with melamine can prevent the agglomeration of $\mathrm{MoS}_{2}$, which can improve the dispersibility of $\mathrm{MoS}_{2}$ in EP resin. Additionally, the amine group of melamine could promote the ring-opening reaction of the EP ring and lead to form a cross-linked structure. Thus, the attached melamine enhanced the interfacial interaction between the $\mathrm{MoS}_{2}$ and EP matrix. Simultaneously, because the melamine and $\mathrm{MoS}_{2}$ were widely used flame retardant, the flame retardance of EP resin will be improved. Therefore, this work provides a facile way to produce of high-performance EP nanocomposites.

${ }^{a}$ School of Materials Science and Engineering, Shenyang University of Chemical Technology, Shenyang 110142, China

${ }^{b}$ School of Chemistry \& Chemical Engineering, Anhui University of Technology, China. E-mail:polyhx@ciac.ac.cn

'Department of Polymer Science and Engineering, Kyungpook National University, South Korea.E-mail: kbyoon@knu.ac.kr

$\dagger$ Electronic supplementary information (ESI) available. See DOI: $10.1039 / \mathrm{c} 8 \mathrm{ra} 02689 \mathrm{k}$

\section{Experimental}

\section{Materials}

Molybdenum disulfide $\left(\mathrm{MoS}_{2}, \sim 6 \mu \mathrm{m}\right), n$-butyllithium $(2.5 \mathrm{M}$ in hexane), melamine (99\%), polypropylenglycol diglycidyl ether 
(PPGDGE, $M_{\mathrm{n}} \approx 640$ ) and polyoxypropylene diamine (D230, $M_{\mathrm{n}}$ $\approx 640$ ) were purchased from Sigma-Aldrich and used as received. EP resin (EPON 828) was obtained from Shell.

\section{Preparation of organophilic $\mathrm{MoS}_{2}$}

$\mathrm{MoS}_{2}$ was first exfoliated according to a literature method. ${ }^{42}$ Typically, $5 \mathrm{~g} \mathrm{MoS}_{2}$ was placed in an autoclave, and $20 \mathrm{~mL} n$ butyllithium (2.5 $\mathrm{M}$ in hexanes) was added. The autoclave was heated at $90{ }^{\circ} \mathrm{C}$ for $12 \mathrm{~h}$ under stirring. After that, the product was filtered and washed with anhydrous hexane $(5 \times 100 \mathrm{~mL})$. The resultant lithium intercalated $\mathrm{MoS}_{2}$ was vacuum-dried and immersed in melamine aqueous solution ( $3 \mathrm{~g}$ in $1000 \mathrm{~mL} \mathrm{H}_{2} \mathrm{O}$ ) under ultrasonication for $4 \mathrm{~h}$ to produce a colloidal suspension of melamine-functionalized $\mathrm{MoS}_{2}\left(\mathrm{M}-\mathrm{MoS}_{2}\right)$. The suspension was neutralized with $1 \mathrm{M} \mathrm{HCl}$, and the products were washed with distilled water $(3 \times 1 \mathrm{~L})$ and then washed with methanol to remove unreacted melamine. Subsequently, $\mathbf{M}-\mathrm{MoS}_{2}$ was obtained by freeze-drying.

\section{Preparation of EP/MoS ${ }_{2}$ nanocomposites}

The desired amount of $\mathrm{MoS}_{2}$ or $\mathbf{M}-\mathrm{MoS}_{2}$ powder was dispersed in appropriate amount of acetone and then stirred with a magnetic stirrer at room temperature for $30 \mathrm{~min}$, followed by sonicated for $60 \mathrm{~min}$. After that the mixture was mixed with desired amount of $\mathrm{EP}(m($ EPON828 $) / m($ PPGDGE $)=55 / 45)$ and sonicated for another $60 \mathrm{~min}$ at $50{ }^{\circ} \mathrm{C}$. The acetone was then removed by vacuum distillation under stirring with a magnetic stirrer at $80{ }^{\circ} \mathrm{C}$. When the mixture was cooled down to $50{ }^{\circ} \mathrm{C}$, a stoichiometric amounts of curing agent (D230) corresponding to $100 \%$ of EP resin content was added and stirred for some time. The resulting mixture was then outgassed in a vacuum oven at $60{ }^{\circ} \mathrm{C}$ for a short period of time and then cast into a Teflon mold with special size. The sample was cured at $75{ }^{\circ} \mathrm{C}$ for 2 hours and post-cured at $120{ }^{\circ} \mathrm{C}$ for 8 hours.

\section{Characterization of $\mathrm{EP} / \mathrm{MoS}_{2}$ nanocomposites}

X-ray diffraction (XRD) patterns were obtained on a Germany BRUKER D8 ADVANCE diffractometer with $\mathrm{Cu} \mathrm{K} \alpha \mathrm{X}$-ray radiation. The scanning range was $2-50^{\circ}$ with a scanning speed of $1^{\circ} \min ^{-1}$. The sample specimens for transmission electron microscopy were microtomed with a diamond knife using a Leica Ultramicrotome at liquid $\mathrm{N}_{2}$ atmosphere. TEM images were obtained from a Japan JEOL JEM-1011 microscope operating at $200 \mathrm{kV}$ in bright field mode. Differential scanning calorimeter (DSC) was conducted on Perkin-Elmer Diamond thermal analyzer. The samples were first heated from room temperature to $50{ }^{\circ} \mathrm{C}$ under $\mathrm{N}_{2}$ atmosphere at heating rate of $10{ }^{\circ} \mathrm{C} \min ^{-1}$ and was then cooled to $-50{ }^{\circ} \mathrm{C}$. Finally, the sample was reheated to $50{ }^{\circ} \mathrm{C}$ at $10{ }^{\circ} \mathrm{C} \mathrm{min}^{-1}$. Tensile properties were performed on a USA INSTRON 5869 electronic testing instrument according to ASTM D638 at a cross-head speed of 5 $\mathrm{mm} \mathrm{min}^{-1}$ at room temperature. The dumbbell like specimens $\left(20 \times 4 \times 2 \mathrm{~mm}^{3}\right)$ were cut from the above cured sample casted on Teflon mold. Each tensile value reported is the average of 5 tests. Optical microscope photos were obtained from an optical microscope (ANA-006, Leitz, Germany) and recorded using a charge-coupled device camera.

\section{Results and discussion}

$\mathrm{MoS}_{2}$ was exfoliated according to a literature method and functionalized by melamine. The exfoliation and functionalization process were given in Scheme 1. To confirm the successful functionalization of $\mathrm{MoS}_{2}$, Fourier transform infrared (FTIR) spectroscopy, XRD analysis and thermogravimetric analysis (TGA) were conducted. Fig. 1(a) shows the FTIR spectra of the bulk $\mathrm{MoS}_{2}$, melamine and $\mathrm{M}-\mathrm{MoS}_{2}$. No characteristic peaks appear in the spectrum of bulk $\mathrm{MoS}_{2}$, whereas several sharp peaks are observed for the $\mathrm{M}-\mathrm{MoS}_{2}$ sample. The two new peaks at 3340 and $3120 \mathrm{~cm}^{-1}$ resulting from $-\mathrm{NH}_{2}$ stretching of the melamine, imply the existence of the mela-

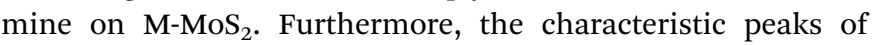
melamine (CN stretching and $\mathrm{NH}_{2}$ bending vibrations) at $1078 \mathrm{~cm}^{-1}$ and $1300-1700 \mathrm{~cm}^{-1}$ were apparently shifted, indicating the formation of covalent bonds between melamine and $\mathrm{MoS}_{2}$. These results indicate that melamine was successfully grafted onto the $\mathrm{MoS}_{2}$ surface.

The bulk $\mathrm{MoS}_{2}$ and M-MoS 2 samples were characterized by XRD. As shown in Fig. 1(b), bulk $\mathrm{MoS}_{2}$ shows a single (002) diffraction peak at $2 \theta=14.3^{\circ}$, which corresponds to a $d$-spacing of $0.6 \mathrm{~nm}$. Upon exfoliation and functionalization, this peak becomes dramatically smaller and broader, and several new peaks are observed at lower $2 \theta$ values. $\mathrm{M}-\mathrm{MoS}_{2}$ shows two new and very broad diffraction peaks at $2 \theta=7.7^{\circ}$ and $9.3^{\circ}$ (corresponding to $d$-spacings of 1.1 and $0.9 \mathrm{~nm}$, respectively), which indicate an increase in the layer distance of $\mathrm{MoS}_{2}$ owing to the graft of melamine. Additionally, the weak and broad diffraction peak also indicate the crystallinity of the $\mathrm{M}-\mathrm{MoS}_{2}$ filler is low.

Thermal stabilities of the bulk $\mathrm{MoS}_{2}$ and $\mathrm{M}-\mathrm{MoS}_{2}$ were investigated by TGA under nitrogen with a temperature range from the room temperature to $800{ }^{\circ} \mathrm{C}$. As presented in Fig. 1(c), bulk $\mathrm{MoS}_{2}$ is clearly very thermally stable, as the mass loss is only $0.8 \mathrm{wt} \%$ upon heating to $800{ }^{\circ} \mathrm{C}$. In contrast, for $\mathrm{M}-\mathrm{MoS}_{2}$ sample, three degradation steps are observed. In the first step $\left(<200{ }^{\circ} \mathrm{C}\right)$, the weight loss is due to the evaporation of physically adsorbed water; in the second step $\left(220-380{ }^{\circ} \mathrm{C}\right)$, the weight loss is caused by decomposition of melamine that is functionalized on the $\mathrm{MoS}_{2}$ surface; and in the third step $\left(>380^{\circ} \mathrm{C}\right)$, the weight loss may be attributed to decomposition of the carbon formed on the $\mathrm{MoS}_{2}$ surface as a result of carbonization of melamine. The weight content of melamine in $\mathbf{M}-\mathrm{MoS}_{2}$ nanofillers was calculated from the char yield of TGA measurement to $\sim 52 \mathrm{wt} \%$. However, the

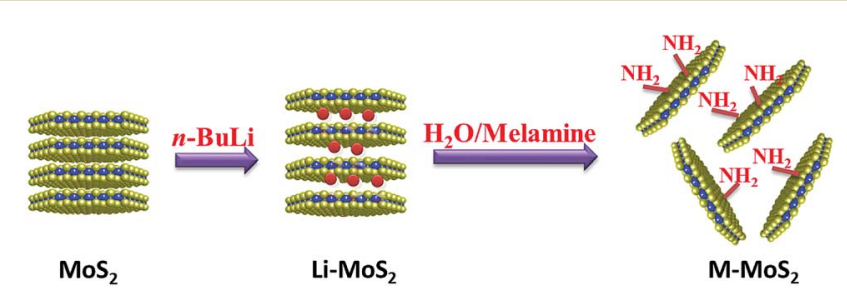

Scheme 1 Preparation of $\mathrm{M}-\mathrm{MoS}_{2}$. 

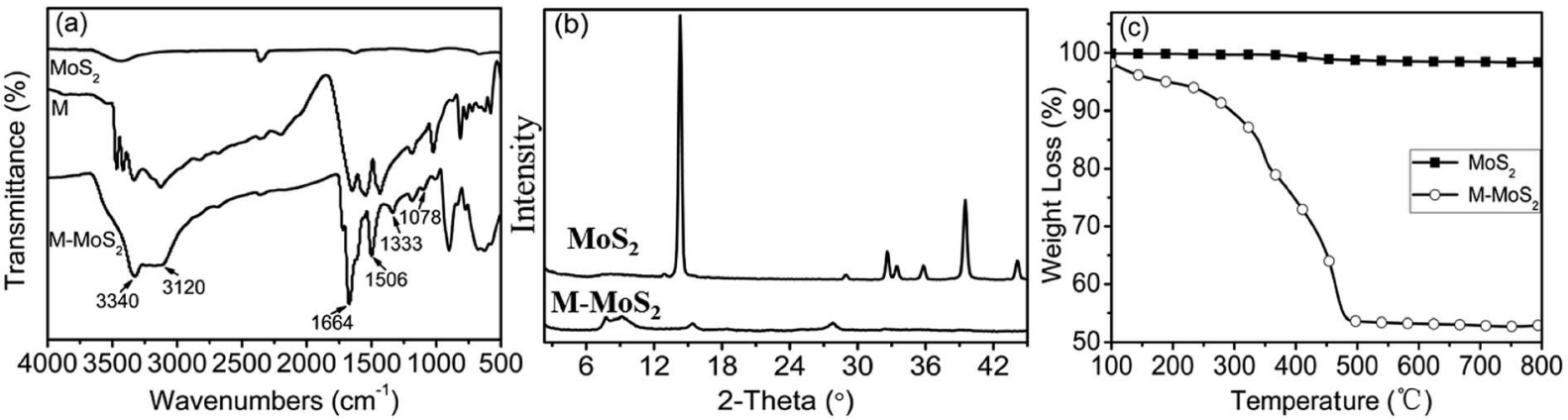

Fig. 1 (a) FTIR spectra, (b) XRD spectra, and (c) TGA curves of $\mathrm{MoS}_{2}$ and $M-\mathrm{MoS}_{2}$.

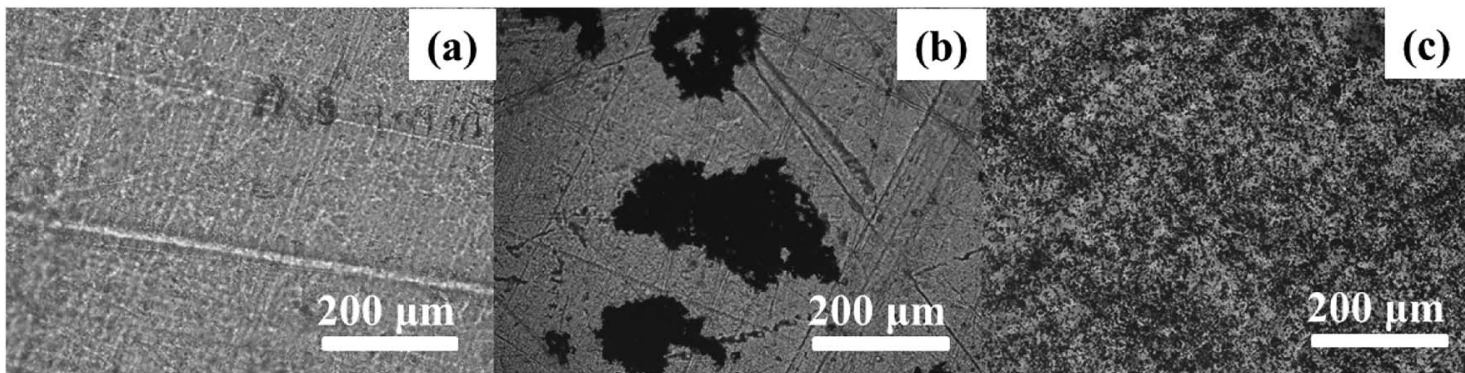

Fig. 2 Optical micrographs of (a) EP, (b) EP/1 wt\% $\mathrm{MoS}_{2}$ and (c) EP/1 wt\% M-MoS 2 nanocomposites.

content of melamine calculated from TGA is not exact, because the $\mathrm{C}_{3} \mathrm{~N}_{4}$ will formed during the carbonization process.

In order to investigate the dispersion of $\mathrm{MoS}_{2}$ and $\mathrm{M}-\mathrm{MoS}_{2}$ in the EP matrix, the resultant thin films of EP, EP/MoS ${ }_{2}$ and EP/M$\mathrm{MoS}_{2}$ nanocomposites were prepared. The thin films were observed under an optical microscope in transparent mode; the obtained micrographs are shown in Fig. 2. It was found that the $\mathrm{M}-\mathrm{MoS}_{2}$ fillers are well dispersed in the EP matrix, while $\mathrm{MoS}_{2}$ aggregation was observed in $\mathrm{EP} / \mathrm{MoS}_{2}$ nanocomposite with $1 \mathrm{wt} \% \mathrm{MoS}_{2}$ addition.

In order to investigate the dispersion and interaction of $\mathrm{MoS}_{2}$ and $\mathrm{M}-\mathrm{MoS}_{2}$ in the EP matrix, the resulted samples were

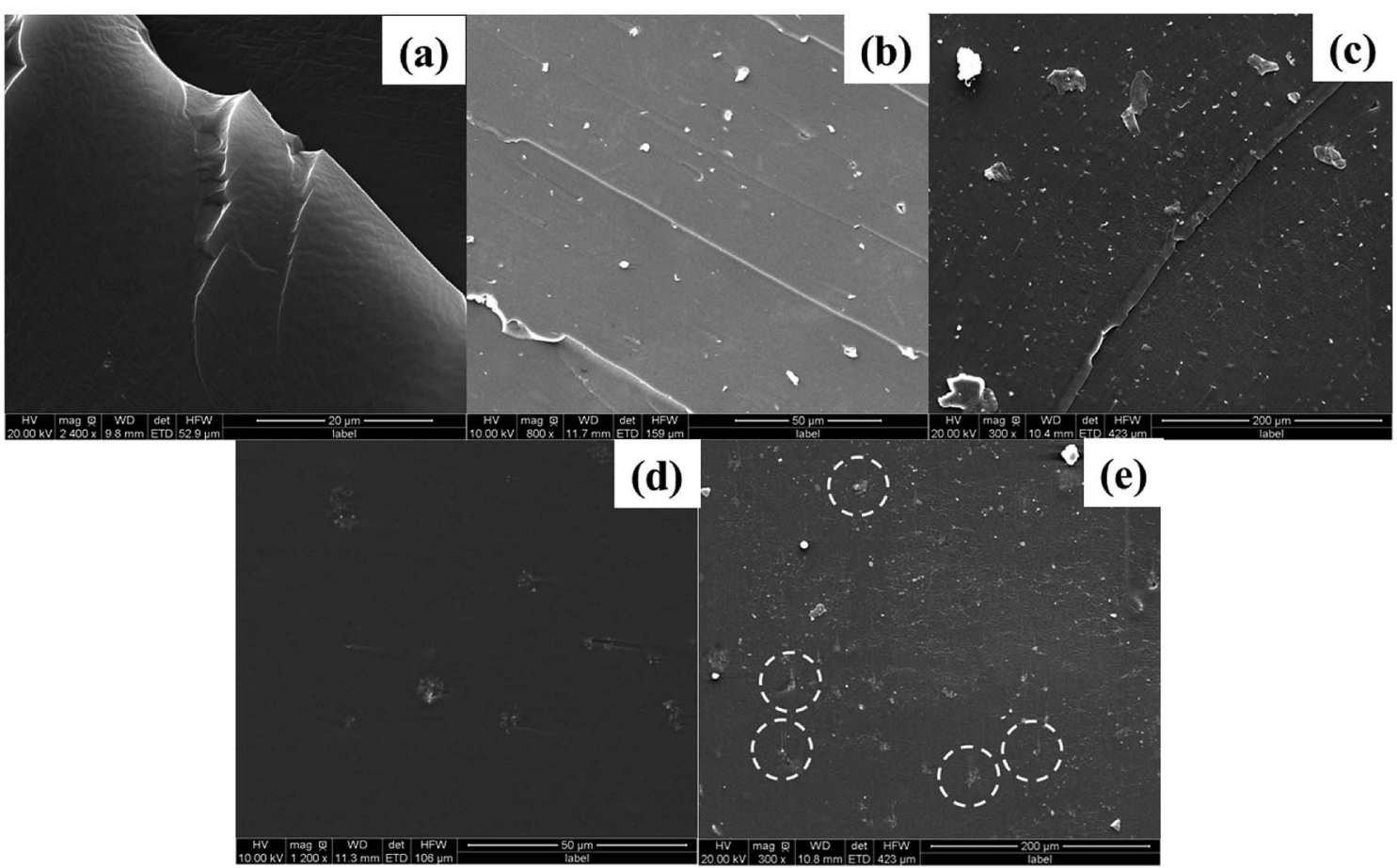

Fig. 3 SEM images of the fractured surface of (a) EP, (b and c) EP/1 wt\% MoS 2 and (d and e) EP/1 wt\% M-MoS 2 nanocomposites. 


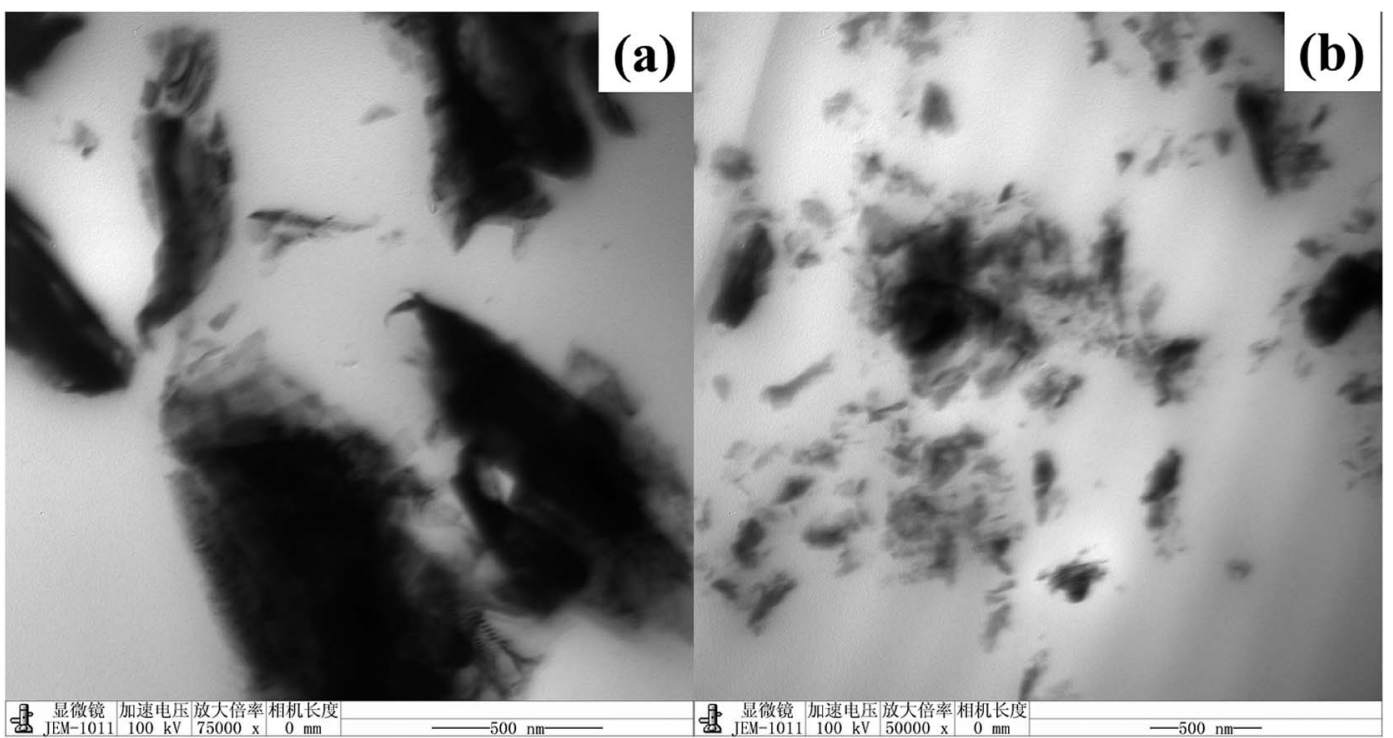

Fig. 4 TEM images of (a) EP/MoS 2 and (b) EP/M-MoS 2 nanocomposite with 1 wt\% filler addition.

characterized by SEM and TEM. As shown in Fig. 3, the SEM image of the $\mathrm{EP} / \mathrm{MoS}_{2}$ nanocomposite exhibited a smooth fractured surface and some $\mathrm{MoS}_{2}$ sheet could be clear observed on the surface of the fractured surface without interaction with EP matrix. With regards to EP/M-MoS 2 nanocomposites, the wrapped structure was observed, implying the strong interaction between $\mathrm{M}-\mathrm{MoS}_{2}$ and the EP matrix. In order to fully characterized the dispersion of fillers in the nanocomposites, TEM of the microtomed section of compression molded samples was examined (Fig. 4). It was found that the $\mathrm{M}-\mathrm{MoS}_{2}$ well dispersed in the EP matrix, while $\mathrm{MoS}_{2}$ aggregation was observed in $\mathrm{EP} / \mathrm{MoS}_{2}$ nanocomposite. This morphology is correlated with the morphology obtained by optical micrographs. We therefore expected the EP/M-MoS 2 nanocomposites will exhibit better mechanical properties than EP nanocomposite with $\mathrm{MoS}_{2}$ fillers.

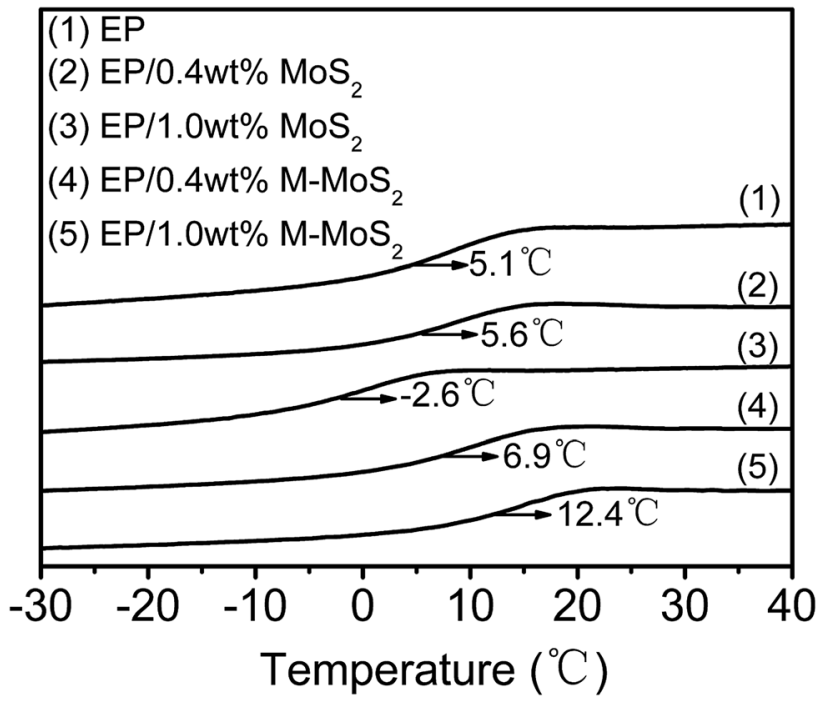

Fig. 5 DSC curve of EP, EP/MoS 2 and EP/M-MoS 2 nanocomposites.
The effect of $\mathrm{MoS}_{2}$ and $\mathrm{M}-\mathrm{MoS}_{2}$ on the glass transition temperature $\left(T_{\mathrm{g}}\right)$ of EP was investigated by DSC; the typical DSC curves are shown in Fig. 5 The $T_{\mathrm{g}}$ of the virgin EP was $5.1{ }^{\circ} \mathrm{C}$. Upon introduction of $\mathrm{MoS}_{2}$, the $T_{\mathrm{g}}$ value tend to decrease with increasing $\mathrm{MoS}_{2}$ content, while the $T_{\mathrm{g}}$ gradually increased with the $\mathrm{M}-\mathrm{MoS}_{2}$ content increasing. With only $1 \mathrm{wt} \% \mathrm{M}-\mathrm{MoS}_{2}$ addition, the $T_{\mathrm{g}}$ of EP rises up to $7.3^{\circ} \mathrm{C}$. The increment of $T_{\mathrm{g}}$ refers to the reduction of matrix chain mobility by the presence of $\mathrm{M}-\mathrm{MoS}_{2}$. During fabrication, melamine molecules bridged $\mathrm{MoS}_{2}$ with EP matrix and a strong interface was thus produced. The strong interface restricts the motion of polymer chain and thus gives rise to the increase in $T_{\mathrm{g}}$. While, with regards to EP/ $\mathrm{MoS}_{2}$ nanocomposites, the reduced $T_{\mathrm{g}}$ are probably caused by two reasons (i) agglomeration when neat $\mathrm{MoS}_{2}$ fillers added. (ii) Reduction of the EP matrix's cross-linking density due to the barrier effect of $\mathrm{MoS}_{2}$.

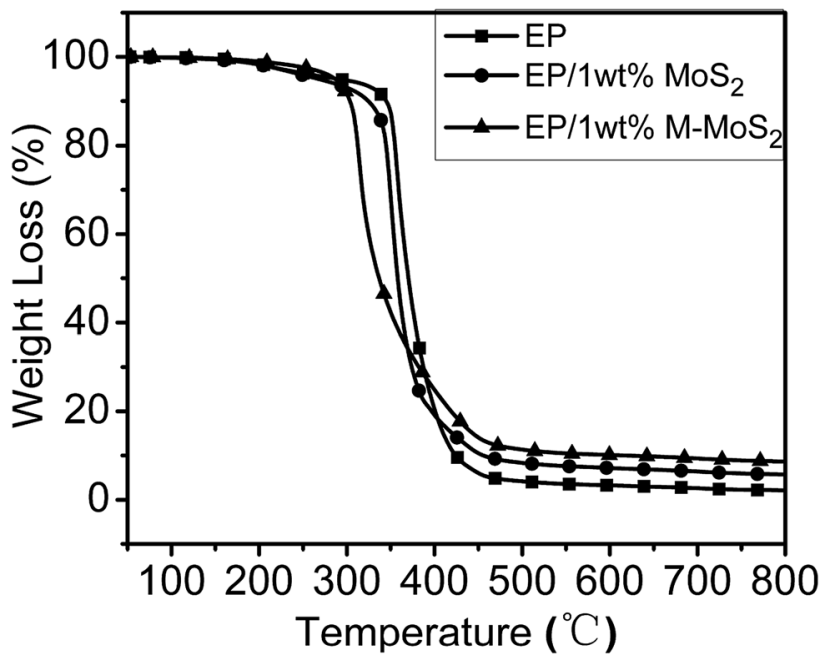

Fig. 6 TGA curves of EP, EP/MoS 2 and $\mathrm{EP} / \mathrm{M}-\mathrm{MoS}_{2}$ nanocomposites. 

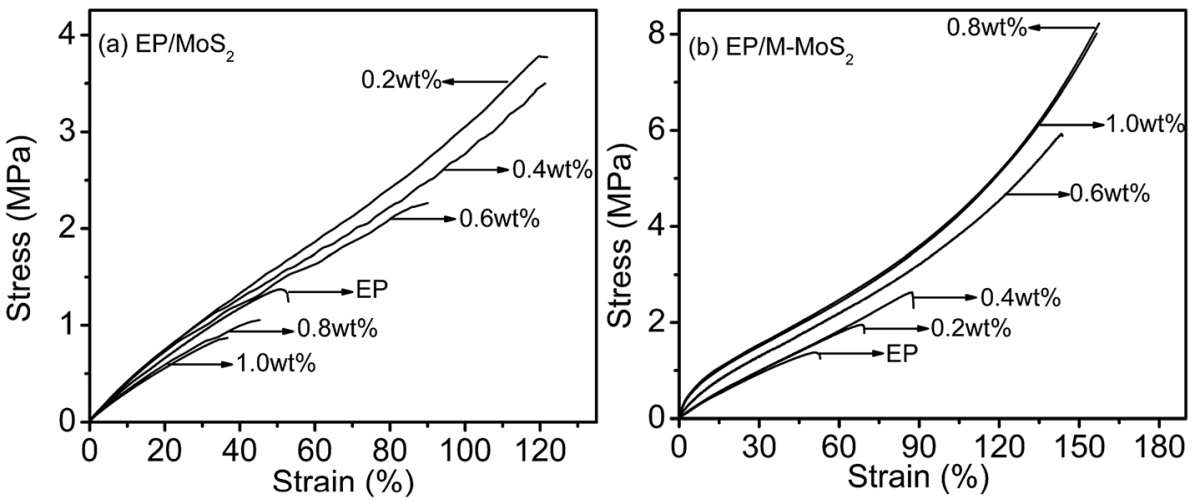

Fig. 7 Stress-strain curves of EP, EP/MoS 2 and EP/M-MoS 2 nanocomposites.

Thermal stabilities of the EP, EP/MoS 2 and EP/M-MoS ${ }_{2}$ were evaluated by TGA under nitrogen atmosphere. As can be observed in Fig. 6, all the nanocomposites present similar degradation behaviors, suggesting that the existence of $\mathrm{MoS}_{2}$ and $\mathrm{M}-\mathrm{MoS}_{2}$ did not significantly affect the degradation mechanism of the matrix polymers. For the EP/MoS 2 and $\mathrm{EP} / \mathrm{M}-\mathrm{MoS}_{2}$ nanocomposites, their degradation temperature is lower than that of pure EP, which could be attributed to the earlier thermal degradation of melamine functional groups on $\mathrm{MoS}_{2}$ surface and/or the high thermal conductivity of $\mathrm{MoS}_{2}$. However, the addition of $\mathrm{MoS}_{2}$ or $\mathrm{M}-\mathrm{MoS}_{2}$ fillers exhibited higher char residues compared to neat EP. Additionally, it can also be seen that the weight loss rates of the EP/M-MoS ${ }_{2}$ nanocomposites was lower than the $\mathrm{EP}$ and $\mathrm{EP} / \mathrm{MoS}_{2}$ nanocomposites. This phenomenon played an important role in improving the flame retardancy of the EP resins. When increasing the temperature, the melamine degraded at first and form char on the $\mathrm{MoS}_{2}$ surface. The formed char can provide a protective shield of mass and heat transfer, which slow down the heat release rate during the thermal degradation process.

The influence of $\mathrm{MoS}_{2}$ and $\mathrm{M}-\mathrm{MoS}_{2}$ on the mechanical properties of the EP nanocomposite is evaluated using a Universal Testing Machine (UTM). The reinforcing effects of the $\mathrm{MoS}_{2}$ and $\mathrm{M}-\mathrm{MoS}_{2}$ on the tensile properties of the EP composites are summarized in Fig. 7 and Table 1. Clearly, the tensile strength and modulus of the resultant $\mathrm{EP} / \mathrm{M}-\mathrm{MoS}_{2}$ nanocomposites were significantly enhanced, even at very low $\mathrm{M}-\mathrm{MoS}_{2}$ nanofiller loadings. The tensile modulus of EP/M-MoS nanocomposites increased from 3.7 to $18.6 \mathrm{MPa}$ (approximately a $400 \%$ increase over neat EP), and the tensile strength increased from 1.5 to $8.3 \mathrm{MPa}$ (approximately a $450 \%$ increase over neat EP) when the $\mathrm{M}-\mathrm{MoS}_{2}$ content increased from 0 to $0.8 \mathrm{wt} \%$. When the $\mathrm{M}-\mathrm{MoS}_{2}$ content higher than $0.8 \mathrm{wt} \%$, the tensile strength and modulus value barely changed. Upon introduction of $\mathrm{MoS}_{2}$, as the $\mathrm{MoS}_{2}$ content increased from 0 to $0.4 \mathrm{wt} \%$, the tensile strength and modulus of $\mathrm{EP} / \mathrm{MoS}_{2}$ nanocomposites slightly increased, but a reducing trend was observed for the further increasing $\mathrm{MoS}_{2}$ content. This phenomenon could be attributed to the agglomerate of $\mathrm{MoS}_{2}$ fillers and reduced the effective contact area between the $\mathrm{MoS}_{2}$ surface and the EP matrix, and thus reduced the reinforcement efficiency. At the same time, melamine as a modifier not only improves the interfacial compatibility between molybdenum disulfide and EP, but also acts as a co-curing agent to promote cross-linking of EP (Scheme 2). Therefore, the addition of $\mathrm{M}-\mathrm{MoS}_{2}$ greatly improves the mechanical properties of EP.

Table 1 Mechanical properties of EP, EP/MoS 2 and EP/M-MoS 2 nanocomposites

\begin{tabular}{|c|c|c|c|c|}
\hline \multirow[t]{4}{*}{$\mathrm{MoS}_{2}$} & 0 & $1.5 \pm 0.2$ & $3.7 \pm 0.2$ & $58.2 \pm 5.5$ \\
\hline & 0.4 & $3.7 \pm 0.4$ & $4.6 \pm 0.7$ & $123.3 \pm 5.8$ \\
\hline & 0.6 & $2.2 \pm 0.1$ & $4.2 \pm 0.4$ & $88.5 \pm 3.5$ \\
\hline & 0.8 & $0.9 \pm 0.1$ & $3.2 \pm 0.2$ & $43.7 \pm 2.6$ \\
\hline & 0.2 & $2.1 \pm 0.2$ & $4.0 \pm 0.1$ & $72.0 \pm 4.2$ \\
\hline & 0.4 & $2.6 \pm 0.2$ & $4.1 \pm 0.3$ & $88.0 \pm 5.0$ \\
\hline & 0.6 & $6.2 \pm 0.3$ & $10.5 \pm 2.7$ & $143.3 \pm 5.8$ \\
\hline & 0.8 & $8.3 \pm 0.1$ & $18.6 \pm 1.2$ & $160.0 \pm 0.0$ \\
\hline & 1.0 & $7.9 \pm 0.3$ & $19.7 \pm 4.3$ & $153.3 \pm 11.5$ \\
\hline
\end{tabular}



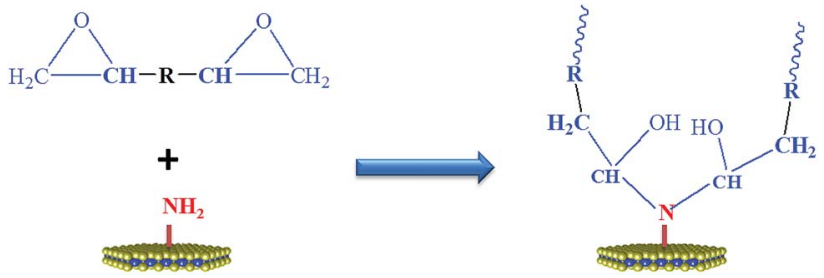

Scheme 2 Reaction between EP and M-MoS 2 .

\section{Conclusions}

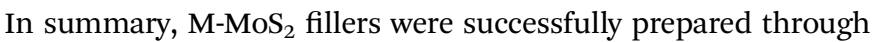
exfoliation of $\mathrm{MoS}_{2}$ followed by reaction with melamine. The effects of $\mathrm{M}-\mathrm{MoS}_{2}$ on the thermal and mechanical properties of EP were investigated. Because the good dispersion and strong interfacial adhesion of $\mathrm{M}-\mathrm{MoS}_{2}$ fillers and the EP matrix, the mechanical properties of EP were significantly improved, even with very low $\mathrm{M}-\mathrm{MoS}_{2}$ addition. Therefore, this work provides a facile way to produce of high-performance EP nanocomposites.

\section{Conflicts of interest}

There are no conflicts to declare.

\section{References}

1 Y. Kang, X. Chen, S. Song, L. Yu and P. Zhang, Appl. Surf. Sci., 2012, 258, 6384-6390.

2 R. A. Gledhill, A. J. Kinloch, S. Yamini and R. J. Young, Polymer, 1978, 19, 574-582.

3 J. Lee and C. Soutis, Compos. Sci. Technol., 2007, 67, 20152026.

4 K. Yoshida, J. Neurophysiol., 2002, 87, 1859-1866.

5 D. L. Zhao, R. H. Qiao, C. Z. Wang and Z. M. Shen, Adv. Mater. Res., 2006, 11-12, 517-520.

6 F. H. Gojny, M. H. G. Wichmann, U. Köpke, B. Fiedler and K. Schulte, Compos. Sci. Technol., 2004, 64, 2363-2371.

7 M. Sangermano, R. A. Ortiz, B. A. P. Urbina, L. B. Duarte, A. E. G. Valdez and R. G. Santos, Eur. Polym. J., 2008, 44, 1046-1052.

8 Z. Diao, Y. Zhao, B. Chen, C. Duan and S. Song, J. Anal. Appl. Pyrolysis, 2013, 104, 618-624.

9 T. Semoto, Y. Tsuji and K. Yoshizawa, Bull. Chem. Soc. Jpn., 2012, 85, 11701-11708.

10 S. Nikafshar, O. Zabihi, M. Ahmadi, A. Mirmohseni, M. Taseidifar and M. Naebe, Materials, 2017, 10, 180.

11 R. Kotsilkova, D. Fragiadakis and P. Pissis, J. Polym. Sci., Part B: Polym. Phys., 2005, 43, 522-533.

12 Q. M. Jia, M. Zheng, C. Z. Xu and H. X. Chen, Polym. Adv. Technol., 2006, 17, 168-173.

13 A. A. Azeez, K. Y. Rhee, S. J. Park and D. Hui, Composites, Part $B, 2013,45,308-320$.

14 M. S. Wang and T. J. Pinnavaia, Chem. Mater., 1994, 6, 468474.
15 C. Kaynak, G. I. Nakas and N. A. IsiTman, Appl. Clay Sci., 2009, 46, 319-324.

16 Y. Ni, S. Zheng, K. Nie, Y. Ni, S. Zheng and K. Nie, Polymer, 2004, 45, 5557-5568.

17 Y. Liu, S. Zheng and K. Nie, Polymer, 2005, 46, 12016-12025.

18 M. Mauro, M. R. Acocella, C. E. Corcione, A. Maffezzoli and G. Guerra, Polymer, 2014, 55, 5612-5615.

19 K. G. Zhou, N. N. Mao, H. X. Wang, Y. Peng and H. L. Zhang, Angew. Chem., 2011, 50, 10839.

20 S. Najmaei, J. Yuan, J. Zhang, P. Ajayan and J. Lou, Acc. Chem. Res., 2015, 48, 31-40.

21 T. Spalvins, J. Vac. Sci. Technol., A, 1987, 5, 212-219.

22 H. U. Kim, H. Kim, C. Ahn, A. Kulkarni, M. Jeon, G. Yeom, M. H. Lee and T. Kim, RSC Adv., 2015, 5, 10134-10138.

23 S. L. Zhang, H. H. Choi, H. Y. Yue and W. C. Yang, Curr. Appl. Phys., 2014, 14, 264-268.

24 H. Hwang, H. Kim and J. Cho, Nano Lett., 2011, 11, 48264830.

25 H. S. Lee, S. W. Min, Y. G. Chang, M. K. Park, T. Nam, H. Kim, J. H. Kim, S. Ryu and S. Im, Nano Lett., 2012, 12, 3695.

26 J. Xie, J. Zhang, S. Li, F. Grote, X. Zhang, H. Zhang, R. Wang, Y. Lei, B. Pan and Y. Xie, J. Am. Chem. Soc., 2013, 135, 17881.

27 J. Xiao, D. Choi, L. Cosimbescu, P. Koech, J. Liu and J. P. Lemmon, Chem. Mater., 2010, 22, 4522-4524.

28 O. Salehzadeh, M. Djavid, N. H. Tran, I. Shih and Z. Mi, Nano Lett., 2015, 15, 5302-5306.

29 J. Lee, Z. Wang, K. He, J. Shan and P. X. L. Feng, ACS Nano, 2013, 7, 6086-6091.

30 C. R. Ryder, J. D. Wood, S. A. Wells and M. C. Hersam, ACS Nano, 2016, 10, 3900.

31 X. Chen and A. R. Mcdonald, Adv. Mater., 2016, 28, 57385746.

32 A. O'Neill, U. Khan and J. N. Coleman, Chem. Mater., 2012, 24, 2414-2421.

33 A. Castellanos-Gomez, M. Poot, G. A. Steele, d. Z. Van, S. J. Herre, N. Agraït and G. Rubio-Bollinger, Adv. Mater., 2012, 24, 772 .

34 K. Zhou, S. Jiang, Y. Shi, J. Liu, B. Wang, Y. Hu and Z. Gui, RSC Adv., 2014, 4, 40170-40180.

35 K. Zhou, J. Liu, B. Wang, Q. Zhang, Y. Shi, S. Jiang, Y. Hu and Z. Gui, Mater. Lett., 2014, 126, 159-161.

36 T. H. Chen, C. Y. Lin, Y. H. Lin, Y. C. Chi, C. H. Cheng, Z. Luo and G. R. Lin, J. Mater. Chem. C, 2016, 4, 9454-9459.

37 H. Zhang, Y. K. Moon, X. Zhang, H. X. Zhang and K. B. Yoon, RSC Adv., 2016, 6, 112429-112434.

38 J. Zhang, H. Feng and B. J. Qiu, Adv. Mater. Res., 2010, 87-88, 345-350.

39 N. Maity, A. Mandal and A. K. Nandi, J. Mater. Chem. C, 2017, 5, 12121-12133.

40 K. Zhou, J. Liu, Y. Shi, S. Jiang, D. Wang, Y. Hu and Z. Gui, ACS Appl. Mater. Interfaces, 2015, 7, 6070.

41 O. Eksik, J. Gao, S. A. Shojaee, A. Thomas, P. Chow, S. F. Bartolucci, D. A. Lucca and N. Koratkar, ACS Nano, 2014, 8, 5282-5289.

42 W. M. Divigalpitiya, R. F. Frindt and S. R. Morrison, Science, 1989, 246, 369-371. 